\title{
Cases study of the effectiveness of ozone gas therapy on the treatment of resistant meningitis in infants with hydrocephalus
}

\begin{abstract}
Hydrocephalus brings great difficulties to Neurosurgeons when it is accompanied with resistant meningitis, because placing a Ventriculo- peritoneal shunt is a contraindication in the presence of meningitis and from the other side quick and safe treatment helps preserve brain's mantel from dystrophy due to hydrocephalus.

We tried a new method (not used before) and it was highly effective against this kind of resistant meningitis - Ozone Therapy Intrathecaly. A five to six sessions of Ozone gas therapy with a dose of $40 \mu \mathrm{g} / \mathrm{ml}$ intrathecaly each other day was able to cure from resistant meningitis in 2 infants with hydrocephalus.
\end{abstract}

Volume 3 Issue 5 - 2015

\author{
Mazen KB Dahhan \\ Vänersborg, Sweden
}

Correspondence: Mazen KB Dahhan, Storgatan 19, Igh: 3, 46236Vänersborg, Sweden, Email mkbd_77@yahoo.com

Received: August 13, 2014 | Published: December 18, 2015

\section{Introduction}

Hydrocephalus brings great difficulties to Neurosurgeons when it is accompanied with resistant meningitis, because placing a Ventriculoperitoneal shunt (the gold standard of treating hydrocephalus in infants) is a contraindication in meningitis and from the other side the need for quick and safe treatment helps preserve the brain's mantel ability from dystrophy due to hydrocephalus

This Hydrocephalus may last for a long time if the shunt is not placed quickly, and there is a need to avoid unnecessary treatments such as external ventricular drainage or repeated CSF evacuation which may bring minimal damage to the brain cortex.

In this study, we present two infants suffering from hydrocephalus, after CSF analysis was done bacterial meningitis was discovered and the bacteria showed high resistance on treatment with usual treatment options, we tried a new method (not used before) and it was highly effective against this kind of resistant meningitis - Ozone Therapy Intrathecaly.

Both cases were treated with antibiotics intrathecally (injected inside dilated ventricles), but because of the resistant nature of the bacteria, cure from meningitis was not achieved, in one case an external shunt drainage was places and intravenicular antibiotics were injected with simultaneous intravenous treatment with other antibiotics.

\section{Material \& methods}

\section{Case I}

Maha Al Jumaa - a 2 months old infant, was presented since birth with communicating Hydrocephalus, as a rule we had to take CSF for analysis before making a decision of shunt placement (Table 1).

Table I A bacterial growth shows growth of gram-negative Bacteria: Pseudomonas Aeruginosa + Staphlococcus Aureus, no sensitivity for over 20 recent antibiotics including $3 r d$ Generation of Cephalosporins

\begin{tabular}{|c|c|c|c|c|c|}
\hline BC & Neu/Lym & RBC & Protein & Glucose & Color \\
\hline 57 & $90 / 10$ & 2500 & 300 & 15 & Yellc \\
\hline
\end{tabular}

The microbial growth analysis was done with the method of (Microgen) with the usage of both Resin and Thioglycolate to cover any unwanted effects from antibiotics that the patient may have taken before. The treatment started with the placement of an external shunt with injection of Gentamycin $10 \mathrm{mg}$ with Amikacin $30 \mathrm{mg}$ daily intrathecaly (although Gentamycin and Amikacin showed no effect on the bacterial incubation - but hopefully they can give some effect as a last chance).

Plus intravenously with Vancomycin $20 \mathrm{mg} / \mathrm{kg}$ in 3 doses and Ceftazidime $50 \mathrm{mg} / \mathrm{kg}$ in 3 doses. (Before the injection of antibiotics into ventricles an amount of about $50 \mathrm{ml}$ of CSF was withdrawn from the ventricles and after that both antibiotics were injected - incase to reduce hydrocephalus and keep the brain mantel status with no tension to improve the general and neurological status of the patient and to increase the concentration of antibiotics within CSF to get full effectiveness).

After 2 weeks CSF Analysis was as follows (Table 2):

Table 2 The external shunt was removed after CSF analysis showed an improvement after 2 weeks of treatment

\begin{tabular}{llllll}
\hline WBC & Neu/Lym & RBC & Protein & Glucose & Color \\
\hline 250 & $90 / 10$ & 1000 & 220 & 20 & Yellow turbid \\
\hline
\end{tabular}

At 3rd week CSF analysis has improved but not to cure from meningitis (Table 3 )

Table 3 CSF analysis was done once weekly, with the bacterial growth test which in week 3 showed not sensitivity also to Imipenem. The above treatment was given for another 2 weeks with transfontanel needle aspiration of CSF and intravenous antibiotical treatment. With fixed results of CSF after 2 weeks from shunt removal we had to search for other treatment options

\begin{tabular}{llllll}
\hline WBC & Neu/Lym & RBC & Protein & Glucose & Color \\
\hline 50 & $95 / 5$ & 250 & 250 & 18 & $\begin{array}{l}\text { Yellow slightly } \\
\text { turbid }\end{array}$
\end{tabular}

The microbial growth analysis was done with the method of (Microgen) with the usage of both Resin and Thioglycolate to cover any unwanted effects from antibiotics that the patient may have taken before. The treatment started with the placement of an external shunt with injection of Gentamycin $10 \mathrm{mg}$ with Amikacin $30 \mathrm{mg}$ daily intrathecaly (although Gentamycin and Amikacin showed no effect on the bacterial incubation - but hopefully they can give some effect as a last chance). 
Plus intravenously with Vancomycin $20 \mathrm{mg} / \mathrm{kg}$ in 3 doses and Ceftazidime $50 \mathrm{mg} / \mathrm{kg}$ in 3 doses. (Before the injection of antibiotics into ventricles an amount of about $50 \mathrm{ml}$ of CSF was withdrawn from the ventricles and after that both antibiotics were injected - incase to reduce hydrocephalus and keep the brain mantel status with no tension to improve the general and neurological status of the patient and to increase the concentration of antibiotics within CSF to get full effectiveness)

After consultation with Dr. S. Kalieh- who has expertise with Ozone gas therapy and certified with this kind of treatment- a suggestion was made after consulting his mentors who said that this treatment wasn't done before, but it was used in many studies for many other diseases including septicemia, hepatitis, MS, ulcers and it was given (Ozone gas) with blood, urine, and it was effective and safe and has a bactericidal, fungicidal and virus-inactivating properties. We searched the literature of Neurosurgery but there was no treatment of meningitis with Ozone.

\section{Ozone Therapy has Effects on:}

\section{Red blood cells: Improvement of rheological properties.}

Activation of red blood cell metabolism with increase of 2, 3-DPG and ATP, plus resultant improvement in $\mathrm{O} 2$ release.

2. Action on immunocompetent cells: moderate activation of mononuclear cells with release of cytokines.

Because there was no other choice left, and the patient had a bad prognosis if not cured and the general and neurological situation was deteriorating, we explained to the parents that this kind of treatment wasn't an option before and we had to take the risk and start treatment with Ozone.

All other antimicrobial treatments were stopped since they showed no effectiveness after 2 weeks.

The decision was made by starting with a low dose of Ozone gas concentration (mixed with pure Oxygen) since results were not clear $20 \mu \mathrm{g} / \mathrm{ml}$ (the safe dose for systemic applications). Then, with higher dose of $40 \mu \mathrm{g} / \mathrm{ml}$ per session each other day.

Ozone Device Company we keep hidden in this article- we can give under request if needed (but it is one of the leading companies in Germany with expertise above 50 years). An amount of $50 \mathrm{ml}$ of CSF was aspirated from the ventricles, a $25 \mathrm{ml}$ was taken away, and the remaining $25 \mathrm{ml}$ was used to be mix with Ozone gas with Pure Oxygen. A Butterfly needle was kept in place while the CSF was mixing with Ozone.

The mixing period was done for about 2 minutes.

Caution!! NO gas shape volume allowed to be injected into ventricles.

Just the Ozone gas needed to be diluted into CSF after shaking the liquid of CSF with Ozone gas gently for a time period about 2 minutes (the color of CSF changes slightly inside the $50 \mathrm{ml}$ syringe). After the second session the CSF shape and color became much better (from near orange color to no color and from turbid to near transparent).

This was confirmed with CSF analysis as following (Table 4):

Table 4 Before the last session CSF was as following (Table 5)

\begin{tabular}{llllll}
\hline WBC & Neu/Lym & RBC & Protein & Glucose & Color \\
\hline 10 & $95 / 5$ & 150 & 110 & 30 & $\begin{array}{l}\text { Near } \\
\text { Transparent }\end{array}$ \\
\hline
\end{tabular}

Table $5 \mathrm{~A}$ period of 7 days was suggested and another CSF analysis was done before surgery (Table 6)

\begin{tabular}{llllll}
\hline WBC & Neu/Lym & RBC & Protein & Glucose & Color \\
\hline 5 & $95 / 5$ & 130 & 85 & 35 & Clear Colorless \\
\hline
\end{tabular}

\section{Table 6}

\begin{tabular}{|c|c|c|c|c|c|}
\hline WBC & Neu/Lym & RBC & Protein & Glucose & Color \\
\hline 0 & & 3 & 75 & 40 & Clear Colorless \\
\hline
\end{tabular}

Five Ozone sessions at each other day manner were done before the complete cure from meningitis: A Ventriculo-Peritoneal shunt was placed and last time the parents were referred after 1 year from the operation with good child neurological status and working shunt device

There was notable development of partial epilepsy after surgery but maybe it was related to the shunt insertion and the surgery itself.

\section{Case 2}

An infant Fatima Mustafa - a 3 month year old, presented with a surgically treated meinigocele 1 month ago And Presented with Hydrocephalus with bacterial meningitis

\section{CSF (Table 7)}

\section{Table 7}

\begin{tabular}{llllll}
\hline WBC & Neu/Lym & RBC & Protein & Glucose & Color \\
\hline 270 & $50 / 50$ & 20 & 320 & 20 & Yellow turbid \\
\hline
\end{tabular}

We had a culture: Klebsiella

But there was no sensitivity to any of the antibiotics, including last generations of Cephalosporins, only Imipenem shoed a ( + one plus) sensitivity on incubation despite no treatment with antibiotics has started before.

The infant treated with Imipenem with the dosage of $25 \mathrm{mg} / \mathrm{kg} \mathrm{q} 6$ hours intravenously and with Gentamycin 10mg and Amikacin 30mg intrathecaly daily.

After 2 weeks of previous intravenous and intrathecal treatment with previous antibiotics we did CSF analysis (Table 8):

Table 8 Treatment with Ozone gas has started at the beginning of the third week after doing the same ethical warning with the parents and after the stable state of meningitis for about 2 weeks confirmed by CSF analysis

\begin{tabular}{llllll}
\hline WBC & Neu/Lym & RBC & Protein & Glucose & Color \\
\hline 210 & $90 / 10$ & 10 & 300 & 25 & Yellow turbid \\
\hline
\end{tabular}

Ozone gas was diluted with CSF and was injected in solution shape with CSF each every day, with started dose of $20 \mu \mathrm{g} / \mathrm{ml}$ of Ozone with pure Oxygen then with $40 \mu \mathrm{g} / \mathrm{ml}$.

After the 5th session CSF was as following (Table 9):

Table 9

\begin{tabular}{llllll}
\hline WBC & Neu/Lym & RBC & Protein & Glucose & Color \\
\hline 8 & $90 / 10$ & 0 & 70 & 40 & Clear Colorless \\
\hline
\end{tabular}

After 1 week of relief we did 6th last session and CSF after that session was done (Table 10):

Table 10

\begin{tabular}{lllll}
\hline WBC & Neu/Lym & RBC & Protein & Glucose \\
\hline 0 & 0 & 60 & 40 & Clor \\
\hline
\end{tabular}


6 sessions of Ozone gas therapy were done before full cure from meningitis

A Ventriculo-Peritoneal shunt was placed, and the patient was seen after 6 month from surgery and she was doing well.

\section{Results}

A five to six sessions of Ozone gas therapy with dose of $40 \mu \mathrm{g} / \mathrm{ml}$ intrathecaly each other day were able to cure from resistant meningitis in 2 infants with hydrocephalus. (With serious and life-threatening infections like Pseudomonas and Klebsiella).

\section{Acknowledgments}

None.

\section{Conflicts of interest}

None.

\section{Conclusion}

1. Ozone gas treatment can be a promising method to treat resistant meningitis in infants with hydrocephalus, but it needs further study and support.

2. Its safety makes it preferable in my cases than placing external shunt drainage, or injecting antibiotics into the ventricles, but its $100 \%$ safety needs to be well studied on many cases.

3. It is an affordable treatment, quick, and available everywhere and can be done in the clinic.

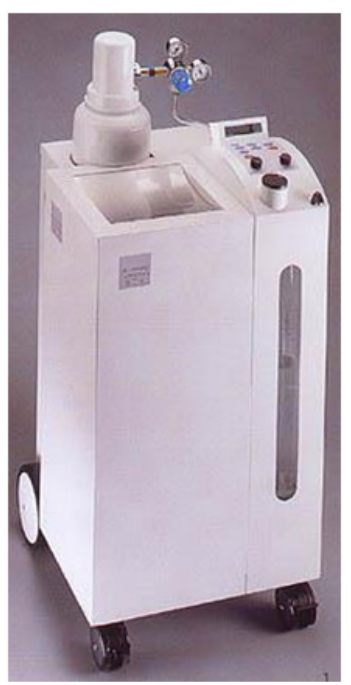

\section{References}

1. Dr. Mazen Dahhan's. Neuro-Spine Surgical private clinic (Patients records), Aleppo, Syria.

2. Al Andalus Hospital (Dr. Kalieh), Aleppo, Ozone Therapy.

3. Center's database.

4. Sabbagh's Laboratory, Aleppo. Dr. Tallah Sabbag, Data of patients.

5. Hänsler VR. Ozone Handbook: Fundamentals - prevention - therapy. Ecomed Verlag, Loseblatt-Sammlung, (A Handbook of Medical Ozone, loose-leaf edition, in German only). 1998.

6. World Health Organization (WHO). Air Quality Guidelines: Global Update 2005. Particulate Matte, Ozone, Nitrogen, Dioxide and Sulfur Dioxide. WHO Regional Office for Europe, Copenhagen, Denmark. 2006. 